\title{
Seeing while acting: hand movements can modulate attentional capture by motion onset
}

\author{
Davood G. Gozli • Jay Pratt
}

Published online: 26 August 2011

(C) Psychonomic Society, Inc. 2011

\begin{abstract}
The onset of new motion has been shown to be a very robust cause of attentional capture, generating a processing advantage for the location of motion onset regardless of the observer's concurrent goal. The present study, motivated by the common-coding account of action and perception, examined whether the effect of motion onset on visual attention can be modulated by the observer's mode of action. Specifically, the commoncoding account predicts that preparing an action can render the features that are used in the action plan less available for visual processes. Consistent with this hypothesis, in Experiment 1 the magnitude of attentional capture caused by a single motion onset was reduced when this motion was similar to the observer's response (i.e., along the same axis). Similarly, in Experiment 2 the onset of a responsedifferent motion gained a processing advantage over the response-similar motion onset when the two were presented simultaneously. Since both types of motion were present in every trial, the results of Experiment 2 suggest that response similarity affected visual-attentional processes rather than motor processes. Together, these results suggest that the processes of attentional prioritization caused by motion onset can be modulated by the observer's concurrent action.
\end{abstract}

Keywords Visual attention - Attentional capture by motion onset - Action planning - Common features for action and perception · Theory of Event Coding

D. G. Gozli $(\bowtie) \cdot$ J. Pratt

Department of Psychology, University of Toronto,

100 St. George Street,

Toronto, ON M5S 3G3, Canada

e-mail: d.gharagozli@utoronto.ca
Visual perception is a selective process, and the visual attentional mechanisms play a crucial role in determining which visual information will be prioritized for more extensive processing. This prioritization is based on the visual properties of stimuli (e.g., Itti \& Koch, 2001; Theeuwes, 1991, 1994) and the goals (e.g., Folk, Remington, \& Johnston, 1992) and actions (e.g., Welsh \& Pratt, 2008; Wykowska, Schubö, \& Hommel, 2009) of the observer. The prioritization of information, which is primarily based on stimulus properties and is independent of any top-down contributions from the observer's goals and actions, is typically referred to as attentional capture. The present study examines an extremely robust form of attentional capture caused by the abrupt onset of motion (Abrams \& Christ, 2003; Al-Aidroos, Guo, \& Pratt, 2010; Franconeri \& Simons, 2003; Guo, Abrams, Moscovitch, \& Pratt, 2010).

In the initial study of attentional capture by motion onset, Abrams and Christ (2003) presented subjects with a set of four figure- 8 placeholders, two in rotational motion and two static, that would later transform into target and distractor letters. The subjects were instructed to identify the target letter ( $\mathrm{S}$ or $\mathrm{H})$ as quickly as possible among the three distractor letters (E's and U's). Critically, at the same time as the placeholders transformed into letters, one of the static placeholders began to move (motion onset), one of the moving placeholders stopped (motion offset), one placeholder continued to move (constant motion), and one placeholder remained stationary (static). Subjects responded faster to target letters when they appeared at the motion onset location rather than at any of the other three locations (Abrams \& Christ, 2003, Exp. 1a), indicating that motion onsets induced capture of attention. Similarly, when a long stimulus onset asynchrony (SOA; Exp. 1b) was used in the same paradigm, more inhibition of return (IOR) was found at the motion onset location than at any other location. 
Abrams and Christ also reported that the processing advantage for the location of motion onset was unaffected by the number of surrounding distractors (Exp. 2). Finding facilitation at a short SOA, inhibition at a long SOA, and insensitivity to the number of distractors are all indications of involuntary attentional capture (e.g., Posner \& Cohen, 1984; Yantis \& Jonides, 1984). Thus, Abrams and Christ concluded that motion onset cues are capable of generating attentional capture (see also Franconeri \& Simons, 2003; Guo et al., 2010).

If the onset of motion captures attention, an important next step is to ask whether this attentional capture can be obtained regardless of the specific task characteristics and the observer's expectations and goals. The influential work of Folk et al. (1992; Folk, Remington, \& Wright, 1994) suggests that when visual targets are defined by a specific feature, a top-down attentional control set is generated that can eliminate attentional capture by salient distractors that do not share the target feature. Importantly, Folk et al. (1994) observed that when a target was defined on the basis of color, an irrelevant motion cue failed to cause attentional capture.

Whether or not capture by motion onset is completely involuntary is still a point of contention (see Al-Aidroos et al., 2010; Cosman \& Vecera, 2010). It is worth noting, for instance, that the motion cues used by Folk et al. (1994) were accompanied by three simultaneous onset cues that may have reduced the salience of motion (Christ \& Abrams, 2008; von Mühlenen, Rempel, \& Enns, 2005). Recently, Al-Aidroos et al. demonstrated that capture by motion onset can occur despite a color-based attentional control set. In that study, subjects looked for color-defined target triangles appearing inside one of two peripheral placeholders. On each trial, either a color cue (which could match or mismatch with the target color) or a motion onset cue occurred at one of the placeholders. As expected, attentional capture was found with matching color cues and not with mismatching color cues. Importantly, quicker responses were also found at motion onset cued locations, indicating that motion onset captured attention despite the fact that subjects' attention was set for a specific color.

Although attentional capture by motion onset seems to resist the top-down influence of the observer's goal (Al-Aidroos et al., 2010), this form of capture may be susceptible to changes in the observer's cognitive state due to the concurrent mode of action (e.g., Bekkering \& Neggers, 2002; Brown, Moore, \& Rosenbaum, 2002; Welsh \& Pratt, 2008; Wykowska et al., 2009). In the present study, we tested whether an observer's action could modulate attentional capture by task-irrelevant motion onset, motivated by the common-coding theory of action and perception (Hommel, Müsseler, Aschersleben, \& Prinz, 2001; Müsseler, 1999; Prinz, 1997). This theory posits that processes underlying action and perception draw on a shared representational domain, such that activation of a particular feature by the action system can affect perceptual processing of that feature occurring at the same time, and vice versa (Hommel et al., 2001).

Evidence for the common-coding account of action and perception comes from findings that show that when an action and a functionally unrelated percept coincide in time and share a common feature, the processing of the action and the processing of the percept interfere with one another (e.g., Kunde \& Wühr, 2004; Müsseler \& Hommel, 1997; Müsseler, Wühr, Danielmeier, \& Zysset, 2005; Schubö, Aschersleben, \& Prinz, 2001; Schubö, Prinz, \& Aschersleben, 2004; Wühr \& Müsseler, 2001; Zwickel, Grosjean, \& Prinz, 2007, 2010). For instance, Müsseler and Hommel examined subjects' perception of briefly presented arrows pointing rightward or leftward while they were making a leftward or rightward manual response. Accuracy was reduced when the arrow and response had the same direction, as compared to when they differed (see also Zwickel et al., 2007).

Further support for the potential interference of actions with visual processing comes from observed systematic changes in the perceived visual feature such that the difference between the action feature and the percept's feature is exaggerated (Schubö et al., 2001, Exp. 4; Zwickel et al., 2010). For example, Schubö et al. (2001) instructed subjects to simultaneously observe a sinusoidal motion presented on the screen (trial $k$ ) and to recreate the motion presented previously (trial $k-1$ ). The authors found that the perceived features (i.e., magnitude and velocity) of motion were "repelled" by the features of the concurrent movement (e.g., performing a small-amplitude and slow-velocity sinusoidal motion caused medium-sized, medium-velocity motions to be perceived with larger magnitude and faster velocity). Similarly, Zwickel et al. (2010) reported that a subject's direction of hand movement can interfere with the perceived direction of a motion stimulus. The exaggerated contrast between the percept and the action also supports the idea of a common representational domain, wherein simultaneous representations of a percept and an action need to remain distinct (Schubö et al., 2001; Schubö et al., 2004; Zwickel et al., 2010).

Given the results supporting the common-coding theory of action and perception (Hommel et al., 2001), it follows that concurrent actions could potentially reduce attentional capture by motion onset by reducing the salience of the new motion. Specifically, in the following experiments, subjects were presented with task-irrelevant motion onset stimuli that either shared or did not share a common feature with a planned movement response. We predicted that capture by motion onset would be selectively reduced or eliminated when the stimulus motion had a feature that was taken up by the action plan. 


\section{Experiment 1}

In the present experiments, we employed a version of the attentional-capture-by-motion-onset paradigm (Abrams \& Christ, 2003; Al-Aidroos et al., 2010; Franconeri \& Simons, 2003; Guo et al., 2010) in which subjects searched for a target letter - with two possible identities - among a set size of four items (i.e., one target and three distractors). The critical addition to the paradigm was that the responses were hand movements (via the computer mouse) along either a horizontal or vertical predetermined axis. Prior to the presentation of the letters, one of the possible target locations was cued by onset of motion along either the horizontal or vertical axis. Thus, axis of motion was the feature that could be shared between the stimulus motion and the response. Whereas the axis of response was set for the entire experimental block, the axis of the motion onset cue randomly varied across the experimental trials. To maintain the functional separation of the stimulus motion and the response, it was important that subjects did not respond to the motion of the item, but rather to the identity of the target letters (Müsseler, 1999). We expected that preparing to make a movement response along one axis would reduce the availability of that axis for perceptual processing of motion, and that this would reduce or eliminate attentional capture by the response-similar motion onset.

\section{Method}

Subjects A group of 24 undergraduate students at the University of Toronto participated in the experiment for course credit. All subjects reported right-handedness and having normal or corrected-to-normal vision.

Apparatus and procedures The experiment was run in a dimly lit and sound-attenuated room, on a Windows-run PC, using MATLAB (The MathWorks, Natick, MA) and the Psychophysics Toolbox (version 3.0.8; Brainard, 1997; Pelli, 1997). The visual stimuli were displayed on a CRT monitor set at $1,024 \times 768$ resolution and a $75-\mathrm{Hz}$ refresh rate. The viewing distance from the monitor was fixed at about $45 \mathrm{~cm}$ with a chin-/headrest. A corded optical computer mouse was used as the response device. The mouse cursor was invisible during the entire task (i.e., the movements of the mouse were not displayed on the screen). The automatic acceleration of the mouse cursor ("enhance pointer precision") was disabled to keep the response criteria (i.e., the movement distance threshold) independent of movement speed. The initial position of the mouse on the table was marked for the subjects. Additionally, four locations were marked on the table around the initial position of the mouse (to the right, left, front, and back), in order to denote both the directions and the optimal distance of movements $(5 \mathrm{~cm})$.

The visual search stimuli (see Fig. 1) were presented in white on a black background. The search stimuli were uppercase letters $\left(0.6^{\circ} \times 1^{\circ}\right)$ presented in Arial font, with E's and U's used as the distractors and $\mathrm{S}$ and $\mathrm{H}$ as the targets. Each letter appeared inside a placeholder, and the four placeholders were white squares $\left(2^{\circ} \times 2^{\circ}\right)$ placed at the corners of an imaginary square $\left(10^{\circ} \times 10^{\circ}\right)$. Thus, the center of each placeholder deviated $7.1^{\circ}$ of visual angle diagonally from the central fixation point.

An example of a trial with a valid motion cue is shown in Fig. 1. Subjects were instructed to begin each trial by looking at the central fixation cross $\left(1^{\circ} \times 1^{\circ}\right)$. After $1,500 \mathrm{~ms}$, one of the placeholders moved with constant speed $\left(0.6^{\circ}\right.$ of displacement per every $\left.20 \mathrm{~ms}\right)$ in one of the four possible directions (upward, downward, leftward, or rightward) to $2.4^{\circ}$ away from its initial position, and then returned to its original position (the entire motion lasted for $160 \mathrm{~ms}$ ). Immediately after, letter stimuli appeared in the placeholders. At any trial, a letter $\mathrm{S}$ or $\mathrm{H}$ appeared along with three distractors (randomly chosen from E's and U's). Subjects were instructed to report the identity of the current target as quickly as possible by moving the mouse cursor in the assigned directions (up or to the right for $\mathrm{S}$, or down or to the left for $\mathrm{H}$, depending on the condition) and then to return the mouse to its initial position in order to prepare for the next trial. Letters remained on the screen until a response was made (i.e., the mouse deviating about $5 \mathrm{~cm}$ from its initial position) or $2,000 \mathrm{~ms}$ had elapsed. Subjects were given feedback when responding incorrectly (MISTAKE!), when responding within the first $100 \mathrm{~ms}$ following search stimulus presentation (TOO QUICK!), or when they did not respond within the first 2,000 ms (TOO LATE!).

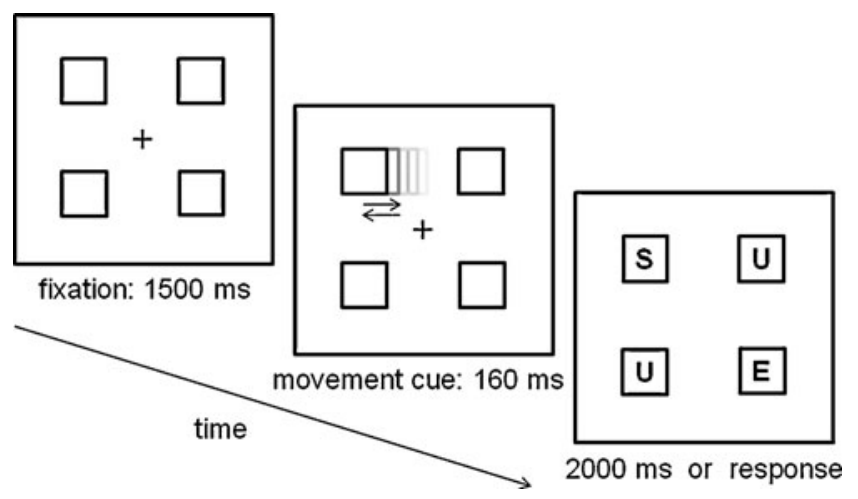

Fig. 1 Progression of events in a trial in Experiment 1. A motion onset (along either the vertical or horizontal axis) cued one location prior to the presentation of letters. The responses were movements on the vertical (up for $\mathrm{S}$, down for $\mathrm{H}$ ) or horizontal (right for $\mathrm{S}$, left for $\mathrm{H}$ ) axis, depending on the block 
Design Each subject performed two experimental blocks, with each block consisting of a practice phase (15 trials) and an experimental phase (256 trials, divided into four segments with brief breaks in between). The target was equally likely to have either of the identities (H vs. S) and to appear in any of the four locations. Similarly, the motion onset cued one of the four locations with $25 \%$ validity. Motion was equally likely to occur at any of the four locations (upper left, upper right, lower left, lower right) and in any of four directions (upward, downward, leftward, or rightward). In counterbalanced blocks, responses were made along either the horizontal axis (right for $\mathrm{S}$, left for $\mathrm{H}$ ) or the vertical axis (up for $\mathrm{S}$, down for $\mathrm{H}$ ).

Results and discussion

Three of the subjects, who had accuracies $3 S D$ s below the mean, were excluded from the analyses. Response times (RTs) from the correct trials were trimmed, excluding responses $2.5 \mathrm{SDs}$ above or below the mean $(3.4 \%$ of trials), and were submitted to a $2 \times 2$ repeated measures ANOVA with cue validity (valid vs. invalid) and S-R axis similarity (same vs. different) as independent factors (see Fig. 2).

The results of the ANOVA showed a main effect of cue validity $\left[F(1,20)=23.6, M S E=767, p<.001, \eta_{\mathrm{p}}{ }^{2}=.542\right]$, with faster responses on validly cued trials $(723 \mathrm{~ms})$ than on invalidly cued trials $(752 \mathrm{~ms})$, indicative of attentional capture by motion onset. No main effect of response-cue similarity was found $(F<1)$. Most importantly, the interaction between cue validity and response-cue similarity reached significance $[F(1,20)=5.2, M S E=281, p<.05$, $\left.\eta_{\mathrm{p}}{ }^{2}=.206\right]$, as the magnitude of attentional capture on S-R axis different trials $(38 \mathrm{~ms})$ was greater than capture on $\mathrm{S}-\mathrm{R}$ axis similar trials $(21 \mathrm{~ms})$.

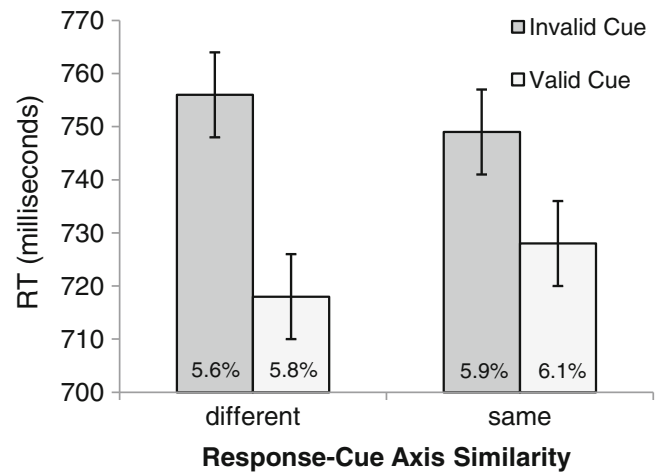

Fig. 2 Mean response times for trials in which the stimulus and response motions were similar (same axis) and different (different axes), for valid and invalid motion onset cues. The error rate for each condition is presented at the base of each graph. Error bars represent 95\% within-subjects confidence intervals (Cousineau, 2005; Loftus \& Masson, 1994)
The error data (see Fig. 2) were submitted to a similar ANOVA, which revealed no significant main effect or interaction of the factors $\left(F_{\mathrm{S}}<1\right)$. Therefore, the RT findings do not seem to be a product of a speed-accuracy trade-off.

These results suggest that attentional capture by motion onset can be reduced when the motion shares a feature with the concurrently planned response. The interaction between cue validity and $\mathrm{S}-\mathrm{R}$ axis similarity is not likely to be caused by motion onsets affecting stimulus-similar responses, because a motor effect would only be consistent with a main effect of S-R similarity. If, for instance, perception of a response-similar motion interfered with planning or execution of the response, we would have found slower responses across both valid and invalid S-R similar conditions. Thus, the lack of a main effect of $\mathrm{S}-\mathrm{R}$ similarity on performance indicates a reduced attentional capture by motion onset.

\section{Experiment 2}

In the previous experiment, preparing a horizontal or vertical movement reduced motion onset capture by cues moving along the same axis. The question addressed in this experiment was whether an action plan can modulate the prioritization among multiple salient motion stimuli. If the reduced attentional capture by S-R similarity (Exp. 1) was due to the differential unavailability of features for visual processing, then if both types of motions were simultaneously presented, the motion onset that was dissimilar to the response should show more attentional capture than the motion onset that was similar to the response.

In addition, this experiment would more conclusively rule out the possibility that the findings of Experiment 1 were based on response modulation. The response modulation account would argue that perceiving different motion onset cues (response-similar vs. response-different) influences the process of planning or executing the manual responses, giving rise to a pattern of findings that resembles modulation of attentional capture. Response modulation, however, would be expected to be spatially nonspecific, and thus would be expected to produce (a) a main effect of S-R axis similarity and (b) no interaction between $\mathrm{S}-\mathrm{R}$ axis similarity and cue validity. Although the results of Experiment 1 were contrary to both expectations, the present experiment tests the response modulation account further, by presenting both types of motion simultaneously in every trial. Thus, any potential effect of the responsesimilar motion on the motor system would be present throughout the experiment, excluding the possibility that response modulation dictated the pattern of results. 
For this experiment, we predicted a relative disadvantage for the location of the response-similar motion onset relative to the location of the response-different motion onset. Thus, the key contrast of interest was between the locations of the response-similar and the response-different cue. To make this contrast more pronounced, the two cues were always presented at maximally distant locations.

\section{Method}

Subjects A group of 26 undergraduate students at the University of Toronto participated in this experiment for course credit. All subjects reported normal or corrected-tonormal vision and previous experience with the computer mouse. The only left-handed subject reported frequent use of the computer mouse with her right hand.

Apparatus and procedure The apparatus and procedures were the same as in Experiment 1, except that in this experiment there were two motion onset cues. The two cues were always orthogonal to each other, in both location and axis of motion. That is, the two cues could either co-occur at the upper left and the lower right locations or at the upper right and the lower left locations. Thus, neighboring placeholders never moved in the same trial. This was done to maximize the competition between the two cues for spatial orienting and to make the comparison between their cuing effects easier. In addition, the two cues always moved in orthogonal axes, such that the response-similar and response-different motion cues were always present within the same trial.

Design Each subject performed two experimental blocks of the same structure as in Experiment 1 (i.e., distinct blocks based on the axis of response). There were three types of trials: (a) trials on which neither of the two cues was valid (invalid), (b) trials on which the response-similar cue was valid (same-axis valid), and (c) trials on which the response-different cue was valid (different-axis valid).

\section{Results and discussion}

Two of the subjects, who had accuracies $3 S D$ s below the mean, were excluded from the analyses. RTs from the correct trials were trimmed, excluding responses $2.5 \mathrm{SDs}$ above or below the mean ( $2.7 \%$ of trials). Our hypothesisdriven comparison between the response-similar and response-different cues (see Fig. 3) revealed a significant difference $[t(23)=2.46, S E=4.17, p<.05]$, due to longer RTs for the response-similar cue $(752 \mathrm{~ms})$ as compared to the response-different cue $(742 \mathrm{~ms})$. Put differently, when comparing both cued locations to the uncued locations, the location of the response-different motion onset was at a

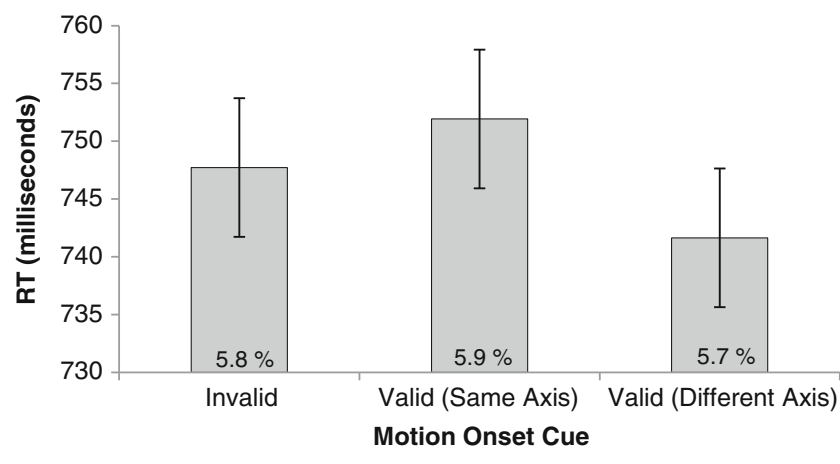

Fig. 3 Mean response times for trials in which the target appeared inside a static placeholder (invalid trials), a placeholder moving along the response axis (valid, same axis), or a placeholder moving perpendicular to the axis of response (valid, different axis). The error rate for each condition is presented at the base of each graph. Error bars represent 95\% within-subjects confidence intervals (Cousineau, 2005; Loftus \& Masson, 1994)

processing advantage (cuing effect: $6 \mathrm{~ms}$ ) relative to the response-different motion onset (cuing effect: $-4 \mathrm{~ms}$ ), although neither of the two types of valid trials was significantly different from invalid trials $(p s>.1)$.

The comparison of error rates between the response-similar $(5.9 \%)$ and response-different $(5.7 \%)$ trials also did not reveal a significant difference $[t(23)=0.56, S E=0.67, p>.5]$, ruling out the possibility of a speed-accuracy trade-off.

The results from this experiment revealed that when a response-similar and a response-different motion onset were presented simultaneously, the response-different motion gained a relative processing advantage over the response-similar motion. This is consistent with the prediction based on the differential availability of motion features for perceptual processes due to response planning. That is, when a hand movement response is prepared on a certain axis (horizontal or vertical), motion along the same axis becomes less available for perceptual processes, leaving the response-similar motion onset at a processing disadvantage.

\section{General discussion}

The present study examined the effect of the observer's mode of action on the attentional prioritization of visual items on the basis of abrupt motion onset (Abrams \& Christ, 2003; Al-Aidroos et al., 2010; Franconeri \& Simons, 2003; Guo et al., 2010). Motivated by the common-coding theoretical account of action and perceptual processes (Hommel et al., 2001; Müsseler, 1999; Prinz, 1997), we hypothesized that preparing an action would decrease the availability of features belonging to the prepared action for visual processes, thus reducing attentional capture by an irrelevant motion that is similar to the 
action. Consistent with our hypothesis, in Experiment 1 we found that the magnitude of capture was significantly smaller on the S-R-similar trials. The selectivity of this disadvantage for S-R similarity was confirmed in Experiment 2, wherein subjects were presented with two simultaneous motion onset cues. Again, responses were slower to the location cued by the response-similar motion onset relative to the location cued by the responsedifferent motion onset. Since both types of motion were present in every trial, Experiment 2 showed that S-R similarity affects visual-attentional processes and not motor processes.

It is worthwhile to contrast the present findings with the somewhat similar recent findings of Cosman and Vecera (2009, 2010), who reported that attentional capture by a task-irrelevant salient feature can be eliminated under high perceptual load. Those modulations, however, are not specific to onsets of a certain motion, or even specific to onset of motion. Whereas performing in a high-load condition seems to have a general effect of attentional capture by salient distractors, our study reports featurespecific modulation of attentional capture by motion onset (see Müsseler, 1999; Zwickel \& Prinz, in press). Without manipulating display complexity and perceptual load across conditions, processing resources were selectively made scarce for motions along one axis due to the similarity to the observer's action, causing a reduction in attentional capture by the onsets of those motions.

Our findings fit well with previous reports on the interference of action with visual perception due to feature overlap (Kunde \& Wühr, 2004; Müsseler \& Hommel, 1997; Müsseler et al., 2005; Schubö et al., 2001, 2004; Wühr \& Müsseler, 2001; Zwickel et al., 2007, 2010). An important aspect of the findings of such interference, which is particularly relevant to our study, is that the overlap of action and perception does not need to be at the lower-level features, but can be a conceptual and symbolic overlap (e.g., verbalizing "left" or making a leftward hand movement reduces perceptual accuracy for a visually presented leftward arrow or the word LEFT; Kunde \& Wühr, 2004). This provides grounds for relying on the similarity between the vertical axis of motion on the computer screen (up vs. down) and the vertical axis of movement for the mouse (forward vs. backward). That is, even though the two "vertical" axes have lower-level feature differences, they overlap conceptually. Our verbal instructions, which described the subjects' mouse movements on the vertical axis as "upward" and "downward," might have been critical to maintaining this conceptual S-R similarity (Hommel, 1993).

A decrease in the salience of motion onset due to the observer's action is consistent with previous reports of reduced perceptual sensitivity for response-similar motion (Zwickel et al., 2007). Our findings, however, do not directly address motion perception as much as the attentional consequences of motion onset. To emphasize the distinct attentional interpretation of our results, it may be useful to view the advantage of a motion onset cue as facilitation in integrating the target features, target location, and response (e.g., Briand \& Klein, 1987; Keizer, Colzato, \& Hommel, 2008; Treisman \& Gelade, 1980). As Briand and Klein (1987, see their Exp. 4) showed, a salient peripheral stimulus particularly facilitates identification of targets that consist of a conjunction of features. Based on this view of attentional orienting, to say that motion onset was less effective in attentional capture is not to say that motion perception was impaired. In fact, the particular motion feature being used in an integrated planned action may still be processed with equal efficiency but remain ineffective at facilitating the binding of the event features (Hommel et al., 2001). This relative ineffectiveness at binding could be viewed as ineffectiveness in attentional capture by the motion cue (Briand \& Klein, 1987; Treisman, 1988, 2006).

To examine the attentional (i.e., effectiveness in feature binding) explanation, we examined changes in the effect of another form of S-R compatibility, based on the relationship between target location and response direction ( $\mathrm{Lu} \&$ Proctor, 1995; Simon, 1990). The location-based S-R compatibility, commonly known as the Simon effect, would speed up responses toward a target location relative to responses away from the target location (e.g., facilitation of a rightward response relative to a leftward response when the target appears to the right of fixation). For responsesimilar trials, the impaired-motion-perception account would not predict any interaction between the Simon effect and cue validity. The ineffective-binding account, on the other hand, would predict that an additional Simon-type compatibility might strengthen the binding of target identity, target location, and response (see Keizer et al., 2008). Thus, performance on trials with valid cues in which binding was relatively inefficient (i.e., S-R axis similar cues) would benefit from the Simon-type compatibility. To test this, data from Experiment 1 were analyzed with a $2 \times$ $2 \times 2$ repeated measures ANOVA using the Simon-type relationship between the target and response (compatible vs. incompatible), S-R motion axis similarity (different vs. similar), and cue validity (valid vs. invalid) as independent factors. Similar to the reported results, this analysis revealed a main effect of cue validity $[F(1,20)=22.9$, $\left.M S E=1,539, p<.001, \eta_{\mathrm{p}}{ }^{2}=.533\right]$, an interaction between cue validity and motion axis similarity $[F(1,20)=5.46$, $\left.M S E=589, p<.05, \eta_{\mathrm{p}}{ }^{2}=.214\right]$, and no main effect of motion axis similarity. In addition, a Simon-type main effect of positional S-R compatibility was found $[F(1,20)=74.0$, $\left.M S E=996, p<.001, \eta_{\mathrm{p}}{ }^{2}=.787\right]$. This positional $\mathrm{S}-\mathrm{R}$ compatibility effect did not interact with $\mathrm{S}-\mathrm{R}$ axis similarity $[F(1,20)=1.66, M S E=734, p=.21]$ or cue validity $(F<1)$. 
It is important to note that the absence of the two-way interaction between axis similarity and positional compatibility, in particular, provides additional evidence against a purely response-based explanation of the primary findings. Finally, the three-way interaction between positional S-R compatibility, motion axis similarity, and cue validity was significant $\left[F(1,20)=4.90, M S E=617, p<.05, \eta_{\mathrm{p}}{ }^{2}=.197\right]$. Examining the cuing effect $\left(\mathrm{RT}_{\text {valid }}-\mathrm{RT}_{\text {invalid }}\right)$ across conditions revealed that, for trials with Simon-type incompatibility, attentional capture by motion onset was completely eliminated for a response-similar motion onset (capture effect $=8 \pm 10 \mathrm{~ms}$ ) while present for the response-different motion onset $(48 \pm 8 \mathrm{~ms})$. For the trials with Simon-type compatibility, however, attentional capture was equal for both response-similar (32 $\pm 9 \mathrm{~ms})$ and response-different (32 \pm $8 \mathrm{~ms}$ ) motion onset cues.

The elimination of attentional capture during trials with Simon-type incompatibility is consistent with the attentional interpretation of the primary findings (i.e., ineffective feature binding instead of degraded motion perception), because it suggests the potential for motion onset cues to facilitate performance (i.e., binding of event features) when they are both valid and at the response-compatible location (see Keizer et al., 2008). On the other hand, when a valid cue moving on the response-similar axis appears at a response-incompatible location, it would not receive the same binding advantage.

A similar analysis of data from Experiment 2 showed that the advantage of the response-different valid cues over the response-similar valid cues was present in the trials with positional S-R incompatibility [cuing advantage $=16 \mathrm{~ms}$; $t(23)=3.35, S E=5, p<.01]$. With positional S-R compatibility, however, response-different cues did not have an advantage over response-similar cues [cuing advantage $=$ $5 \mathrm{~ms} ; t(23)=0.79, S E=6, p>.5]$. Taken together, in both experiments, the response similarity of the axis of motion received a processing disadvantage when the extra binding force of the response code (i.e., the Simon-type compatibility) was absent. This is compatible with our proposed explanation that the response similarity of motion reduced the attentional advantage of the cue by reducing the effectiveness of the cue in facilitating feature binding (Briand \& Klein, 1987; Hommel et al., 2001).

So far, we have assumed that preparing the responses was the source of interference with capture by motion onset. Why do we propose that this is an effect of preparing actions, and not an aftereffect of executed actions? A likely candidate among the aftereffect alternatives would be that motion on trial $k+1$ could be attributed to the subjects' own action on trial $k$, and that this attribution reduced sensitivity to the stimulus motions (e.g., Cardoso-Leite, Mamassian, Schütz-Bosbach, \& Waszak, 2010). There are, however, two reasons that the effects of attentional modulation observed in this study are more likely to be generated by planned responses and not by previously performed responses. First, the temporal delay between the executed action and the cue of the next trial was too long $(1,500 \mathrm{~ms})$ for any self-attribution to occur. Even a $500-\mathrm{ms}$ temporal delay between the felt and the visually observed movements (though short and consistent) has been shown to prevent attributing the visually presented movements to one's own, even when the image actually represents one's own limb (Longo \& Haggard, 2009; Shimada, Hiraki, \& Oda, 2005). The second reason against aftereffect alternatives comes from Wühr and Müsseler (2001), who showed that response-compatible visual stimuli were at a processing disadvantage only when the response was at the planning stage or during execution, but not after the response had been executed (see also Müsseler et al., 2005). Although we suggest that the planned actions are the most likely cause of the attentional modulation, even if they were caused by the aftereffect of actions, our results would still support the general idea that actions can modulate attentional capture by motion onset.

A general note as to why a response-similar percept is inhibited and not facilitated is warranted, not only because finding a facilitation effect for an action-congruent percept may seem more intuitive, but also because of the numerous reports in support of this view (e.g., Bekkering \& Neggers, 2002; Brown et al., 2002; Craighero, Fadiga, Rizzolatti, \& Umiltà, 1999; Symes, Tucker, Ellis, Vainio, \& Ottoboni, 2008; Wohlschläger, 2000; Wykowska et al., 2009). Although all of the factors predicting whether action congruency would facilitate or inhibit perceptual processes have not yet fully been determined, the functional independence of an action and the overlapping visual feature seems to be critical for obtaining inhibition (Müsseler, 1999; Zwickel \& Prinz, in press). Reports of facilitated processing for action-congruent visual features range from faster detection of grasp-congruent objects (e.g., Craighero et al., 1999; Symes et al., 2008), better visual search based on object orientation (Bekkering \& Neggers, 2002) or size (Wykowska et al., 2009) when preparing a grasp, and resolving ambiguous motion direction in a way that is congruent with the direction of hand movement (Wohlschläger, 2000). Importantly, in these studies the feature whose processing was facilitated due to a concurrent action was often a feature on the basis of which visual targets were both identified and acted upon (e.g., target orientation while grasping, Bekkering \& Neggers, 2002; target size or luminance while grasping or pointing, Wykowska et al., 2009). Due to the association of both the action and the target visual feature with the task, the two remain functionally linked, causing the action plan to facilitate congruent perceptual processing through attentional weighting of features (Wykowska et al., 2009; Zwickel \& Prinz, in press). In the present task, however, responses were made on the 
basis of letter identity, with motion onset being a taskirrelevant, though still salient, feature. Ensuring the functional independence of the action and the visual feature was, therefore, a critical factor for obtaining the inhibition effect (Zwickel \& Prinz, in press).

To summarize, the significance of our findings is twofold. First, they relate to the growing body of work on contextualizing vision within the ongoing demands of the action system. Previous work has suggested that a change in the mode of action can cause different treatments of visual features such as onset (Welsh \& Pratt, 2008), luminance and size (Wykowska et al., 2009), orientation (Bekkering \& Neggers, 2002), and binocular disparity (Brown et al., 2002) in visual attentional processes. Here, we put forth the novel observation that action can cause different treatment of motion onset in a manner that is feature-specific. Second, our findings have implications for the nature of feature representation. Specifically, through examining how visual prioritization of motion onset can be affected by action, we offer further empirical support for the view that the cognitive representations underlying action and perception are shared between the two types of events (Hommel et al., 2001; Müsseler, 1999; Prinz, 1997).

Author Note We thank Adriane Seiffert, Bernhard Hommel, and two anonymous reviewers for their helpful comments. We also thank Bianca Lemus Lavarreda, Pamela Leung, and Amy Nathan for their help with data collection. This project was funded by a discovery grant from the Natural Sciences and Engineering Council of Canada (NSERC) to J.P. and an Ontario Graduate Scholarship to D.G.G.

\section{References}

Abrams, R. A., \& Christ, S. E. (2003). Motion onset captures attention. Psychological Science, 14, 427-432. doi:10.1111/14679280.01458

Al-Aidroos, N., Guo, R. M., \& Pratt, J. (2010). You can't stop new motion: Attentional capture despite a control set for colour. Visual Cognition, 18, 859-880. doi:10.1080/13506280903343085

Bekkering, H., \& Neggers, S. F. W. (2002). Visual search is modulated by action intentions. Psychological Science, 13, 370-374. doi:10.1111/j.0956-7976.2002.00466.x

Brainard, D. H. (1997). The psychophysics toolbox. Spatial Vision, 10, 433-436. doi:10.1163/156856897X00357

Briand, K. A., \& Klein, R. M. (1987). Is Posner's "beam” the same as Treisman's "glue"? On the relation between visual orienting and feature integration theory. Journal of Experimental Psychology. Human Perception and Performance, 13, 228-241. doi:10.1037/ 0096-1523.13.2.228

Brown, L. E., Moore, C. M., \& Rosenbaum, D. A. (2002). Featurespecific perceptual processing dissociates action from recognition. Journal of Experimental Psychology. Human Perception and Performance, 28, 1330-1344. doi:10.1037/0096-1523.28.6.1330

Cardoso-Leite, P., Mamassian, P., Schütz-Bosbach, S., \& Waszak, F. (2010). A new look at sensory attenuation: Action-effect anticipation affects sensitivity, not response bias. Psychological Science, 21, 1740-1745. doi:10.1177/0956797610389187
Christ, S. E., \& Abrams, R. A. (2008). The attentional influence of new objects and new motion. Journal of Vision, 8(3), 27:1-8. doi: $10.1167 / 8.3 .27$

Cosman, J. D., \& Vecera, S. P. (2009). Perceptual load modulates attentional capture by abrupt onsets. Psychonomic Bulletin \& Review, 16, 404-410. doi:10.3758/PBR.16.2.404

Cosman, J. D., \& Vecera, S. P. (2010). Attentional capture by motion onsets is modulated by perceptual load. Attention, Perception, \& Psychophysics, 72, 2096-2105. doi:10.3758/APP.72.8.2096

Cousineau, D. (2005). Confidence intervals in within-subject designs: A simpler solution to Loftus and Masson's method. Tutorials in Quantitative Methods for Psychology, 1, 42-45.

Craighero, L., Fadiga, L., Rizzolatti, G., \& Umiltà, C. (1999). Action for perception: A motor-visual attentional effect. Journal of Experimental Psychology. Human Perception and Performance, 25, 1673-1692. doi:10.1037/0096-1523.25.6.1673

Folk, C. L., Remington, R. W., \& Johnston, J. C. (1992). Involuntary covert orienting is contingent on attentional control settings. Journal of Experimental Psychology. Human Perception and Performance, 18, 1030-1044. doi:10.1037/0096-1523.18.4.1030

Folk, C. L., Remington, R. W., \& Wright, J. H. (1994). The structure of attentional control: Contingent attentional capture by apparent motion, abrupt onset, and color. Journal of Experimental Psychology. Human Perception and Performance, 20, 317-329. doi:10.1037/0096-1523.20.2.317

Franconeri, S. L., \& Simons, D. J. (2003). Moving and looming stimuli capture attention. Perception \& Psychophysics, 65, 999-1010. doi:10.3758/BF03194829

Guo, R. M., Abrams, R. A., Moscovitch, M., \& Pratt, J. (2010). Isoluminant motion onset captures attention. Attention, Perception, \& Psychophysics, 72, 1311-1316. doi:10.3758/APP.72.5.1311

Hommel, B. (1993). Inverting the Simon effect by intention: Determinants of direction and extent of effects of irrelevant spatial information. Psychological Research, 55, 270-279. doi:10.1007/BF00419687

Hommel, B., Müsseler, J., Aschersleben, G., \& Prinz, W. (2001). The Theory of Event Coding (TEC): A framework for perception and action planning. The Behavioral and Brain Sciences, 24, 849-878. doi:10.1017/S0140525X01000103

Itti, L., \& Koch, C. (2001). Computational modelling of visual attention. Nature Reviews Neuroscience, 2, 194-203. doi:10.1038/35058500

Keizer, A. W., Colzato, L. S., \& Hommel, B. (2008). Integrating faces, houses, motion, and action: Spontaneous binding across ventral and dorsal processing streams. Acta Psychologica, 127, 177-185. doi:10.1016/j.actpsy.2007.04.003

Kunde, W., \& Wühr, P. (2004). Actions blind to conceptually overlapping stimuli. Psychological Research, 68, 199-207. doi:10.1007/s00426-003-0156-3

Loftus, G. R., \& Masson, M. E. J. (1994). Using confidence intervals in within-subject designs. Psychonomic Bulletin \& Review, 1, 476-490. doi:10.3758/BF03210951

Longo, M. R., \& Haggard, P. (2009). Sense of agency primes manual motor responses. Perception, 38, 69-78. doi:10.1068/p6045

Lu, C.-H., \& Proctor, R. W. (1995). The influence of irrelevant location information on performance: A review of the Simon and spatial Stroop effects. Psychonomic Bulletin \& Review, 2, 174-207. doi:10.3758/BF03210959

Müsseler, J. (1999). How independent from action control is perception? An event-coding account for more equally-ranked crosstalks. In G. Aschersleben, T. Bachmann, \& J. Müsseler (Eds.), Cognitive contributions to the perception of spatial and temporal events (Advances in Psychology (pp, Vol. 129, pp. 121147). Amsterdam: Elsevier.

Müsseler, J., \& Hommel, B. (1997). Blindness to response-compatible stimuli. Journal of Experimental Psychology. Human Perception and Performance, 23, 861-872. doi:10.1037/0096-1523.23.3.861 
Müsseler, J., Wühr, P., Danielmeier, C., \& Zysset, S. (2005). Actioninduced blindness with lateralized stimuli and responses. Experimental Brain Research, 160, 214-222. doi:10.1007/s00221-0042009-8

Pelli, D. G. (1997). The VideoToolbox software for visual psychophysics: Transforming numbers into movies. Spatial Vision, 10, 437-442. doi:10.1163/156856897X00366

Posner, M. I., \& Cohen, Y. (1984). Components of visual orienting. In H. Bouma \& D. G. Bouwhuis (Eds.), Attention and performance $X$ : Control of language processes (pp. 531-556). Hillsdale: Erlbaum.

Prinz, W. (1997). Perception and action planning. European Journal of Cognitive Psychology, 9, 129-154. doi:10.1080/713752551

Schubö, A., Aschersleben, G., \& Prinz, W. (2001). Interactions between perception and action in a reaction task with overlapping S-R assignments. Psychological Research, 65, 145-157. doi: $10.1007 / \mathrm{s} 004260100061$

Schubö, A., Prinz, W., \& Aschersleben, G. (2004). Perceiving while acting: Action affects perception. Psychological Research, 68, 208-215. doi:10.1007/s00426-003-0133-x

Shimada, S., Hiraki, K., \& Oda, I. (2005). The parietal role in the sense of self-ownership with temporal discrepancy between visual and proprioceptive feedbacks. NeuroImage, 24, 12251232. doi:10.1016/j.neuroimage.2004.10.039

Simon, J. R. (1990). The effects of an irrelevant directional cue on human information processing. In R. W. Proctor \& T. G. Reeve (Eds.), Stimulus-response compatibility: An integrated perspective (pp. 31-86). Amsterdam: North-Holland.

Symes, E., Tucker, M., Ellis, R., Vainio, L., \& Ottoboni, G. (2008). Grasp preparation improves change detection for congruent objects. Journal of Experimental Psychology. Human Perception and Performance, 34, 854-871. doi:10.1037/00961523.34.4.854

Theeuwes, J. (1991). Cross-dimensional perceptual selectivity. Perception \& Psychophysics, 50, 184-193. doi:10.3758/BF03212219

Theeuwes, J. (1994). Stimulus-driven capture and attentional set: Selective search for color and visual abrupt onsets. Journal of Experimental Psychology. Human Perception and Performance, 20, 799-806. doi:10.1037/0096-1523.20.4.799
Treisman, A. (1988). Features and objects: The fourteenth Bartlett memorial lecture. Quarterly Journal of Experimental Psychology, 40A, 201-237. doi:10.1080/02724988843000104

Treisman, A. (2006). How the deployment of attention determines what we see. Visual Cognition, 14, 411-443. doi:10.1080/ 13506280500195250

Treisman, A. M., \& Gelade, G. (1980). A feature-integration theory of attention. Cognitive Psychology, 12, 97-136. doi:10.1016/00100285(80)90005-5

von Mühlenen, A., Rempel, M. I., \& Enns, J. T. (2005). Unique temporal change is the key to attentional capture. Psychological Science, 16, 979-986. doi:10.1111/j.1467-9280.2005.01647.x

Welsh, T. N., \& Pratt, J. (2008). Actions modulate attentional capture. Quarterly Journal of Experimental Psychology, 61, 968-976. doi:10.1080/17470210801943960

Wohlschläger, A. (2000). Visual motion priming by invisible actions. Vision Research, 40, 925-930. doi:10.1016/S0042-6989(99)00239-4

Wühr, P., \& Müsseler, J. (2001). Time course of the blindness to response-compatible stimuli. Journal of Experimental Psychology. Human Perception and Performance, 27, 1260-1270. doi:10.1037/ 0096-1523.27.5.1260

Wykowska, A., Schubö, A., \& Hommel, B. (2009). How you move is what you see: Action planning biases selection in visual search. Journal of Experimental Psychology. Human Perception and Performance, 35, 1755-1769. doi:10.1037/a0016798

Yantis, S., \& Jonides, J. (1984). Abrupt visual onsets and selective attention: Evidence from visual search. Journal of Experimental Psychology. Human Perception and Performance, 10, 601-621. doi:10.1037/0096-1523.10.5.601

Zwickel, J., Grosjean, M., \& Prinz, W. (2007). Seeing while moving: Measuring the online influence of action on perception. Quarterly Journal of Experimental Psychology, 60, 1063-1071. doi:10.1080/17470210701288722

Zwickel, J., Grosjean, M., \& Prinz, W. (2010). On interference effects in concurrent perception and action. Psychological Research, 74, 152-171. doi:10.1007/s00426-009-0226-2

Zwickel, J., \& Prinz, W. (in press). Assimilation and contrast: The two sides of specific interference between action and perception. Psychological Research. doi:10.1007/s00426-011-0338-3 